\title{
Apuntes sobre la construcción del concepto de Jesarrollo en América Latina y su contribución en los procesos de integración en la región
}

\section{Remarks on the construction of the concept of development in Latin America and its contribution to the regional integration process}

Adriana Montenegro-Braz

Facultad Latinoamericana de Ciencias Sociales

Ecuador

Recibido: 29/3/2017 Aceptado: 17/7/2017

\section{Resumen}

La idea de desarrollo ha estado ligada a las discusiones teóricas en torno a si es el Estado o el mercado los que pueden responder a los problemas del crecimiento. Bajo este enfoque, este trabajo tiene como objetivo revisar cómo ha evolucionado la idea de desarrollo en América Latina y su discusión en el regionalismo latinoamericano. Por lo tanto, se realiza un recuento que inicia con el enfoque estructuralista y la teoría de la dependencia de los años cincuenta y sesenta. Luego, se analizan los cambios de la región en los años noventa a partir de

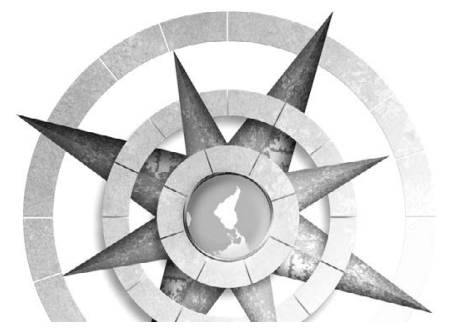

la aplicación de las políticas neoliberales.

Por último, se analizan las nuevas tendencias de integración en la región. En ese sentido, pese a la heterogeneidad de los procesos de integración actuales, el fin de la convergencia neoliberal permitió plantear nuevamente la idea de desarrollo en la región.

Palabras clave: desarrollo, regionalismo, estructuralismo, teoría de la dependencia regionalismo abierto, regionalismo posliberal, América Latina.

\section{Abstract}

The idea of development has been associated with theoretical discussions about whether the State or the market can respond to the problems of growth. According to this approach, this paper aims to research how the idea of development in Latin America and its discussion in the Latin-American regionalism has evolved. Therefore, our recount begins with the structuralist approach and the dependency theory 
of the 50's and 60's. Then, the changes undergone by the region in the 90's with the implementation of neoliberal policies are addressed to finally analyze the new trends of integration in the region. In this sense, despite the heterogeneity of the current integration processes, the end of neoliberal convergence made it possible to once again raise the idea of development in the region.

Keywords: development, regionalism, structuralism, dependency theory, open regionalism, post-liberal regionalism, Latin America

\section{Introducción}

La idea de desarrollo es uno de los conceptos más antiguos de la lógica occidental, generalmente entendido como un proceso direccional, cumulativo e irreversible del crecimiento. Sin embargo, desde la modernidad este concepto ha estado ligado a la idea de progreso, en donde las principales discusiones teóricas han girado en torno a si es el Estado, más asociado con las teorías marxistas, o el mercado, desde las posturas liberales y neoliberales, los que pueden dar una respuesta a los problemas del crecimiento (Hettne, 1995, pp. 29-34).

Esta discusión en un mundo globalizado ha tomado otros matices, pues se ha observado que el Estado no puede dar una respuesta por si solo a los desafíos que se le presentan en el mundo actual. Es por esta razón, que los procesos de regionalización se han intensificado en los últimos años; sin embargo, sigue existiendo esta puja entre lo económico o lo político como respuesta a los problemas de desarrollo en el nivel internacional.

Ahora bien, estas reflexiones no han pasado desapercibidas en el debate que sobre este tema se ha dado en América Latina $(\mathrm{AL})$. Más aún, se ha observado que durante los últimos sesenta años de discusión sobre el desarrollo en la región, se han alternado períodos en donde se ha apostado por dar un mayor peso a lo político, entendido este como una mayor presencia del Estado en el nivel interno y externo, o al capital, en donde se confía en las fuerzas del mercado para equilibrar los posibles desajustes económicos.

Bajo este enfoque, este trabajo tiene como objetivo revisar cómo ha ido evolucionando la idea de desarrollo en América Latina y cómo esta idea permeó, o no, en los modelos de regionalismo. Por lo tanto, este trabajo realizará un recuento que inicia con el enfoque estructuralista en la década de los cincuenta, en donde se observa la construcción de un pensamiento propio sobre el desarrollo que dio paso a esquemas de integración con una fuerte presencia estatal que

32 Apuntes sobre la construcción del concepto de desarrollo en América Latina y su contribución en los procesos de integración en la región 
tenían como propósito implementar modelos orientados a proteger la industria nacional, promover la producción y mejorar los esquemas de complementariedad en la región.

Posteriormente, se pasará a revisar la teoría de la dependencia en donde si bien las críticas al concepto de desarrollo tuvieron una escasa aplicación en la práctica, sin embargo, al igual que la teoría estructuralista, permitieron cuestionar los paradigmas etnocéntricos y construir las bases de un pensamiento latinoamericano en la materia. Luego, se observarán los cambios que se dieron en la región en los años ochenta y noventa, periodo que se caracteriza por la aplicación de políticas neoliberales sin una agenda de desarrollo definida, todo lo cual tuvo repercusiones en el tipo de regionalismo por el que se apostó en aquellas décadas.

Por último, se analizará las nuevas tendencias de integración en la región y la toma de distancia que algunos de esos procesos han realizado frente a las posturas neoliberales. En ese sentido, se observará que la narrativa neoliberal en América Latina ha perdido su hegemonía y eso ha permitido volver a plantear al desarrollo como idea central en los procesos de integración.

\section{El estructuralismo, su propuesta de regionalismo autonómico y su visión de desarrollo para la región}

Luego de la Segunda Guerra Mundial se inició un debate sobre el desarrollo, especialmente por los impactos que habían generado las dos grandes guerras en el seno de Europa y en el resto del mundo. Con todo, parte de la discusión abarcaba también los problemas de "subdesarrollo" no solo en los países que se estaban formando a partir de los procesos de descolonización, sino también en América Latina que no había conseguido alcanzar el tan famoso "despegue" económico ${ }^{1}$. Sin embargo, el hecho de que la subdisciplina de la economía del desarrollo tuviera una serie de contratiempos para explicar los problemas que se estaban dando en los países "menos desarrollados", hacía necesario encontrar una receta que emanara de estos países, evitando así los sesgos etnocéntricos (Hettne, 1995, pp. 35-39).

1 El despegue o take off fue el término utilizado por el economista norteamericano Walt Rostow, a finales de los cincuenta y principios de los sesenta, para describir una de las principales etapas del desarrollo que van desde la sociedad tradicional a la sociedad de consumo masivo. Para este economista es en la etapa de despegue en donde todo los obstáculos para el desarrollo económico son removidos (Hettne, 1995, pp. 52-53).
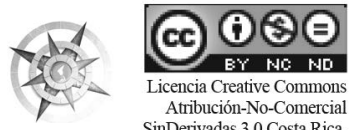

icencia Creative Commons Atribución-No-Comercial
SinDerivadas 3.0 Costa Rica.
Apuntes sobre la construcción del concepto de desarrollo en América Latina y su contribución en los procesos de integración en la región Adriana Montenegro-Braz 
El estructuralismo en América Latina tuvo como base tres textos fundacionales en los que Raúl Prebisch (CEPAL 1951a; 1951b; Prebisch, 1973) no solo estableció los principales lineamientos del enfoque, sino que además orientó teórica e ideológicamente a la Comisión Económica para América Latina y el Caribe (CEPAL) en su primera etapa ${ }^{2}$ (Bielschowsky, 2009 , p. 175). Cabe recalcar que el enfoque cepalino se alejó de concepciones económicas ortodoxas, pues no consideraban al subdesarrollo como una etapa previa al desarrollo, sino más bien como parte de un proceso global que se producía por un intercambio desigual entre los países. Sin embargo, el estructuralismo no planteó salir del sistema capitalista, sino que consideraba que era necesario realizar modificaciones estructurales en las economías de los países en desarrollo para lograr una mayor productividad y acumulación de riquezas (Dos Santos, 1998; Hettne, 1995, pp. 28-29; Zona Económica, 2015).

Ahora bien, bajo esta consideración la CEPAL se preocupó especialmente por la difusión internacional del progreso técnico y la distribución de sus beneficios, una vez que había

2 La CEPAL tuvo dos etapas en su pensamiento, una que va de 1948 a 1990, a la que denominaron etapa estructuralista, y de 1990 a 2008 que se llamó etapa neoestructuralista (Bielschowsky, 2009, pp. 173-184). observado que existía una desigualdad de los ingresos entre los países primario exportadores y los países con una mayor industrialización (Prebisch, 1996, p. 1078). A este respecto, el autor observó que la especialización en la producción de bienes primarios en América Latina tuvo como resultado un deterioro de los términos del intercambio que provocaba déficits macroestructurales en las economías latinoamericanas ${ }^{3}$ (Prebisch, 1996, pp.1078-1079; Bielschowsky, 2009, p. 175, Lorenzini, 2014, p. 17).

Sin embargo, este deterioro de las condiciones del intercambio no podían ser contrarrestadas fácilmente; de hecho, pese a que la industrialización en algunos países de América Latina ${ }^{4}$ se había iniciado por los efectos de la recesión de los años treinta y la Segunda Guerra Mundial, no se evidenciaba el fortalecimiento y desarrollo de sus economías (Bielschowsky, 2009, p. 175; Cardoso y

3 En otras palabras, una elasticidad baja de la demanda mundial de productos primarios, y la consiguiente disminución de sus precios, así como un aumento en los países periféricos de la demanda de los productos industrializados del centro, provocaba déficits crónicos en las balanzas comerciales de los países de (AL), inflación y endeudamiento externo (Prebisch, 1996, pp. 1078-1079; Bielschowsky, 2009, p. 175; Lorenzini, 2014, p. 17).

4 En especial países como Argentina, México, Chile, Colombia y Brasil (Cardoso y Faletto, 1971, p. 4).

34 Apuntes sobre la construcción del concepto de desarrollo en América Latina y su contribución en los procesos de integración en la región 
Faletto, 1971, p. 3). La explicación para este fenómeno, según Prebisch, consistía en que $(\mathrm{AL})$ formaba parte de un sistema de relaciones económicas internacionales caracterizadas por la existencia de un centro y una periferia, en donde los países industrializados (centro), apoyados en su progreso técnico previo, organizaban el sistema internacional para que sirviera a sus propios intereses. Esto, en última instancia relegaba los países de la periferia a una condición permanente de primario exportadores en función de las necesidades y demandas de los países industrializados (Prebisch, 1996, pp. 1078-1079).

Esta condición de subordinación de la periferia a los países del centro se podía explicar con base en tres características. En primer lugar, además de la especialización en la producción de bienes primarios, existía una baja diversidad productiva, que era el resultado de la falta de inversión en nuevos sectores, debido justamente a la escasez de divisas y ahorros que generaba la especialización en productos no manufacturados. Por otra parte, la heterogeneidad estructural, es decir la coexistencia de actividades tecnológicamente modernizadas con otras atrasadas, proporcionaba un excedente del ingreso muy reducido. Finalmente, el atraso de la estructura institucional (Estado, sector agrícola y empresarial, etc.) tuvo como resultado una capacidad fiscal baja, desperdicios del excedente en inversiones improductivas y consumo suntuario por parte de las élites, todo lo cual se traducía en una baja inversión y avance tecnológico (Bielschowsky, 2009, pp. 175-176, Lorenzini y Pereyra, 2013, pp. 13-14).

Para superar esta condición de periferia era necesario entonces modificar el patrón de desarrollo hacia fuera y apostar por una estrategia de industrialización por sustitución de importaciones (ISI). No obstante, dicha estrategia debería estar estimulada por una intervención estatal planificada y dinámica de la economía, la cual debería incluir una política gubernamental centrada en la inversión de la infraestructura, la reducción de las vulnerabilidades externas, un balance entre los diferentes sectores de la producción y la regulación de la participación del capital extranjero (Prebisch, 1996, pp. 1081-1083; Palacio, 2006).

Por último, la CEPAL recomendaba que para reforzar el modelo ISI era necesaria la creación de un mercado común latinoamericano que ofreciera a las industrias de los países de la periferia un mercado ampliado y las ventajas de la especialización, la complementariedad y las economías de 
escala en el nivel regional. Este modelo de integración económica, que se ha denominado integración hacia adentro, viejo regionalismo ${ }^{5}$ o regionalismo autonómico, proponía un bloque abierto hacia la región, pero cerrado hacia el mercado internacional, con el fin de proteger a las nuevas industrias hasta que pudieran competir en igualdad de condiciones con los productos de los países del centro. Estos presupuestos fueron los que en última instancia inspiraron los lineamientos de la Asociación Latinoamericana de Libre Comercio (ALALC) que nació a comienzos de la década de los sesenta y que congregó en un primer momento a Argentina, Brasil, México, Uruguay, Paraguay, Chile y Perú ${ }^{6}$ (Lorenzini, 2014, p. 17; Palacio, 2006; Briceño, s.f., p. 27).

Ahora bien, el modelo de sustitución de importaciones, y su propuesta de regionalismo cerrado, fueron funcionales por un tiempo limitado. Para comienzos de la década de los años sesenta se observó que se habían generado algunos inconvenientes en las balanzas de pagos de los países

5 En contraposición con el nuevo regionalismo que se produjo a partir del fin de la Guerra Fría y que veremos más adelante.

6 La (ALALC) nace aproximadamente un año y medio después de la firma del Tratado de Montevideo del 18 de febrero de 1960, en donde además de los países mencionados se les unieron posteriormente Ecuador, Colombia, Bolivia y Venezuela (Estay, 2000, p. 53). de la región, especialmente porque el crecimiento industrial necesitaba para fomentar la producción una serie de bienes de capital que debían ser importados, creando de esta manera una dependencia financiera y tecnológica con los países del centro. Además, debido a las grandes desigualdades en la distribución del ingreso en $\mathrm{AL}$, la demanda de manufacturas se redujo a una pequeña élite, con lo que se copaba el mercado fácilmente (Hettne, 1995, p. 93; Palacio, 2006).

Por su parte, si bien en los primeros años de la ALALC se aumentó el comercio entre sus miembros, existió una serie de problemas que mermaron su dinamismo. En general, el grueso de las desgravaciones de la ALALC se realizaron en los primeros años, y en aquellos bienes que no generaban resistencia. Luego, se observó una ralentización del proceso en 1969 , cuando se tuvo que postergar por siete años la constitución de la zona de libre comercio, que estaba planificada en una primera instancia para mediados de 1973; meta que finalmente nunca llegó a darse. Asimismo, la creación del Grupo Andino, a finales de los sesenta, evidenció una incapacidad para asegurar una distribución equitativa de las ganancias en la ALALC, lo que en última instancia llevó a Bolivia,

36 Apuntes sobre la construcción del concepto de desarrollo en América Latina y su contribución en los procesos de integración en la región

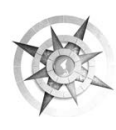


Ecuador, Perú, Colombia y Chile a la conformación de este acuerdo subregional (Estay, 2000, pp. 55-56; Lorenzini y Pereyra, 2013, p. 15).

Por último, todos estos impases hicieron palpable la necesidad de una restructuración de la ALALC, hasta que en 1980 se la transformó en la Asociación Latinoamericana de Integración (ALADI). Lamentablemente, esta transformación tampoco trajo el dinamismo esperado porque a la par de la falta de claridad de plazos y metas, se aunó el hecho de que se plantearon objetivos más ambiciosos que tampoco llegaron a concretarse, como el establecimiento de un mercado común entre los miembros. Esto sin contar con que la crisis de la deuda en el año 82, los conflictos geopolíticos, la inestabilidad de las economías y el deterioro del comercio de la región, complicaron aún más los intentos de crear una integración económica desde una lógica latinoamericana (Estay, 2000, p. 56; Palacio, 2006).

Finalmente, los problemas macroeconómicos en la región ${ }^{7}$, la dificultad para fortalecer la ALALC y avanzar en los acuerdos pactados, así

7 Que se iniciaron en los sesenta, pero que se hicieron más palpables en las décadas de 1970 y posteriormente con la crisis de la deuda de los ochenta. como el surgimiento y difusión de la teoría de la dependencia, llevaron a ver que las propuestas de la CEPAL se estaban agotando (Palacio, 2006; Cardoso y Faletto, 1971, pp. 1-10; Lorenzini, 2014, p. 17).

\section{La teoría de la dependencia y su crítica al paradigma etnocéntri- co de desarrollo}

La principal crítica que le hacía la teoría de la dependencia al estructuralismo de la CEPAL, consistía en que no era suficiente explicar el subdesarrollo en AL con base en argumentos puramente economicistas, dado que el componente político y social del análisis era fundamental. Más aún, la teoría de la dependencia cuestionaba que el desarrollo fuera un proceso incremental, y más bien consideraba que tanto el desarrollo como el subdesarrollo eran dos caras de una misma moneda (Cardoso y Faletto, 1971, p. 11; Hettne, 1995, p. 93, Dos Santos, 1998).

[...] la situación de subdesarrollo se produjo históricamente cuando la expansión del capitalismo comercial y luego del capitalismo industrial vinculó a un mismo mercado economías que, además de presentar grados diversos de diferenciación del sistema productivo, pasaron a ocupar posiciones distintas de la estructura 
global del sistema capitalista. De ahí que entre las economías desarrolladas y subdesarrolladas no sólo exista una simple diferenciación de etapa o de estado del sistema productivo, sino también de función o posición dentro de una misma estructura económica internacional de producción y distribución. Ello supone, por otro lado, una estructura definida de relaciones de dominación (Cardoso y Faletto, 1971, p. 23).

Con todo, la teoría de la dependencia no tiene una postura homogénea. Existen varias vertientes que si bien comparten la idea principal de la interrelación entre desarrollo y subdesarrollo en el sistema capitalista mundial, difieren en relación con el énfasis, posición ideológica e, incluso, respecto a las posibles soluciones. Se puede decir que la teoría de la dependencia se divide entre las posturas neomarxistas, que promueven una salida al sistema capitalista, en las que se ubica a Theotonio Dos Santos, Vania Bambirra y Mauro Marini; y, las posturas marxistas más ortodoxas que aceptan el papel del desarrollo del capital, aunque sin la necesidad de alcanzar el desarrollo socialista, en las que se incluyen a Fernando Enrique Cardoso y Enzo
Faletto $^{8}$ (Dos Santos, 1998; Lorenzini, 2014).

Pese a ser una teoría con un importante elemento transformador y con una visión no etnocéntrica del desarrollo, la teoría de la dependencia no generó un impacto en la práctica, tanto al interior de los Estados como en sus procesos de integración. No obstante, es necesario matizar esta crítica pues se puede observar que la teoría de la dependencia tuvo una fuerte influencia, al igual que la teoría estructuralista, en la construcción de un pensamiento latinoamericano en la materia. Además, se debe recalcar el peso que esta teoría ejerció en el debate académico en otras regiones del mundo y en las posturas de los países de la periferia frente al orden económico imperante. Especialmente, se puede citar la incorporación de algunas visiones de la teoría de la dependencia en las peticiones para un Nuevo Orden Económico Internacional (NOEI) planteado en el seno de las Naciones Unidas en 1974 (Hettne, 1995, pp. 102-103).

8 Esta división es una síntesis de la clasificación realizada por Dos Santos, quien no solo ofrece una tipificación en mayor detalle en su texto La teoría de la dependencia un balance histórico y teórico, sino que también cita la clasificación realizada por Gunder Frank (Frank, 1991 citado por Dos Santos, 1998).

38 Apuntes sobre la construcción del concepto de desarrollo en América Latina y su contribución en los procesos de integración en la región 


\section{El regionalismo abierto y la falta de una agenda para el desarrollo}

La propuesta de regionalismo abierto o nuevo regionalismo se dio desde una perspectiva basada en el libre mercado y de apertura del comercio. Varios fueron los factores globales y regionales que contribuyeron a modificar la agenda de desarrollo en América Latina. Desde el punto de vista mundial, se puede citar a la globalización económica y el final de la Guerra Fría como los principales acontecimientos que propiciaron la elaboración de una estrategia con miras a mejorar la inserción internacional de los países de la región (Ibañez, 1999, pp.5-8; Sanahuja, 2007, pp. 75-78).

La globalización económica trajo consigo una serie de cambios tecnológicos que reorganizaron toda la estructura de producción, distribución y consumo, al igual que la ampliación geoeconómica de los mercados. Por otro lado, el fin de la Guerra Fría relajó los condicionantes geopolíticos que dividían al mundo en dos bloques, haciendo que la lógica de la competencia interestatal migre del plano securitista al económico, además de imponer ideológicamente al capitalismo como la única vía posible (Ibañez, 1999, p. 5; Sanahuja, 2007, pp. 75-76; Antxon, 2010, pp. 4-5).
En relación con los condicionantes regionales, hay que destacar que para dar paso a esta nueva oleada de expansión del capitalismo se impuso desde los años ochenta la liberalización de los mercados, la desregulación económica y la falta de control de flujos financieros, que se visibilizó en AL a través de la aplicación de las recetas del Consenso de Washington. Por tal razón, el modelo ISI y el regionalismo cerrado fueron rechazados por haber "aislado" a las economías de la región del comercio mundial (Williamson, 1995, pp. 6786, Sanahuja, 2007, p. 77; Ibañez, 1999, pp. 5-7; Briceño, s.f., p. 24).

Además, el estancamiento de las negociaciones del Acuerdo General sobre Aranceles Aduaneros y Comercio (GATT por sus siglas en inglés) en la Ronda Uruguay hizo que Estados Unidos apostara por el comercio intrarregional a través de propuestas como la Iniciativa para las Américas, y posteriormente el Área de Libre Comercio para las Américas (ALCA). Por último, la aparición de la Unión Europea en 1992 planteó una serie de inquietudes para la región, como el hecho de que este nuevo bloque económico podría transformarse en una "fortaleza", temor que si bien resultó infundado, motivó una reflexión sobre los efectos de la integración y 
su papel para coadyuvar a la promoción del libre comercio y la competitividad internacional (Williamson, 1995, pp. 67-86; Sanahuja, 2007, p. 77; Ibañez, 1999, pp. 5-7).

Por todas estas razones, se apostó por el regionalismo abierto con el fin de mejorar la inserción internacional de los países latinoamericanos en el mercado internacional, incrementar su influencia en las organizaciones multilaterales y promover una mejor gestión de la interdependencia regional. Ahora bien, entre las principales características del esquema de integración abierto se puede citar su enfoque de no discriminación frente a terceros. Es decir, la idea era conformar mercados regionales que si bien mantuvieran un margen preferencial entre los miembros, permitieran la liberalización multilateral del comercio con el fin de lograr que los productos de la región compitieran internacionalmente (Briceño, s.f., p. 26; Sanahuja, 2007, pp. 75-76).

Por otra parte, el nuevo regionalismo tiene como objetivo fomentar una integración más allá de la simple circulación de bienes y servicios, incluyendo temas como "las inversiones, propiedad intelectual, las compras gubernamentales y las normas laborales y ambientales". Esta integración más profunda no solo abarcaba una agenda con mayores elementos, sino que también se asumían compromisos mayores a los acordados en el seno de la Organización Mundial del Comercio (OMC), lo que se denomina como los "OMC Plus" (Briceño, s.f., p. 26).

De igual manera, el regionalismo abierto enfatiza en la participación de la inversión extranjera directa (IED), con el fin de que las empresas extraregionales puedan aportar capitales y tecnologías para los países de la región. Por último, este tipo de regionalismo también se caracterizó por la proliferación de los acuerdos norte-sur que vinculan economías con gran disparidad económica, sin mayores protecciones como un trato especial y diferenciado para los países de menor desarrollo (Briceño, s.f., p. 26; Palacio, 2006).

Incluso, cabe recalcar que la CEPAL tuvo en esta época una transformación sustantiva de sus propuestas que se vio reflejada en su estrategia de "transformación productiva con equidad" lanzada en 19949; la cual propuso también un regionalismo abierto, pero desde una visión más moderada. No obstante, en la

9 Para mayor información sobre la propuesta de la CEPAL, consultar el documento antes mencionado, disponible en: http://archivo.cepal. org/pdfs/1991/S9181285.pdf (CEPAL, 1996).

40 Apuntes sobre la construcción del concepto de desarrollo en América Latina y su contribución en los procesos de integración en la región 
práctica los países de AL asimilaron las posturas de corte neoliberal a tal punto que algunos autores llegan a afirmar que se utilizó a la integración económica para regionalizar el ajuste estructural. Entre los acuerdos de integración que se formaron o reformularon bajo el enfoque de regionalismo abierto podemos citar a los Tratados de Libre Comercio de América del Norte (TLCAN), el Mercado Común del Sur (MERCOSUR) ${ }^{10}$, la transformación del Pacto Andino en la Comunidad Andina (CAN) y el Grupo de los tres (G3) ${ }^{11}$ (Briceño, s.f., p. 27; Briceño, 2003, p. 142 citado por Palacio, 2006; Sanahuja, 2007, p. 78; Ibañez, 1999, p. 8).

En los más de veinte años de regionalismo abierto no se logró el dinamismo prometido y han persistido varias disfuncionalidades. Primero, si bien se lograron establecer zonas de libre comercio en todos los esquemas de integración, persisten aún varias excepciones en rubros importantes, $y$ las trabas no arancelarias continúan siendo un freno para el libre comercio. Ello sin contar con el incumplimiento de los aranceles externos

10 No obstante algunas iniciativas como el MERCOSUR están girando en la actualidad hacia acuerdos de carácter más social (ver Briceño, s.f., p. 24).

11 El Grupo de los tres es un Tratado de Libre Comercio firmado entre México, Colombia y Venezuela en 1994. comunes (AEC) fijados entre los Países miembros. Además, a pesar de la amplia liberalización entre los bloques, el comercio intrarregional permaneció marginal en comparación con el total de exportaciones de cada país $^{12}$ (Sanahuja, 2007, pp. 82-83; Sanahuja, 2012, p. 27).

Por otro lado, hay que recalcar que el regionalismo abierto no ha logrado una armonización macroeconómica en la región, pese a que durante la década de los noventa su interés era la aplicación más o menos ortodoxa, dependiendo del país, del Consenso de Washington. Tampoco se ha construido una normativa común entre los miembros ${ }^{13}$, o las que existen no se aplican o se aplican de manera laxa. Otro punto que se puede citar, es el hecho de que las políticas sectoriales en materias como innovación, ciencia, tecnología, infraestructura o energía, no han avanzado, o los resultados son pequeños, quedando la mayor parte del discurso en mera retórica (Sanahuja, 2007, pp. 83-84).

12 Se dice que el comercio intrarregional ha fluctuado entre un $12 \%$ y $20 \%$ del comercio total, siendo que EUA continúa siendo principal socio de la región (llegando a un $60 \%$ ), aunque su participación ha decaído en los últimos años (ubicándose en un $40 \%$ del comercio total (Durán y Lo Turco, 2010, p. 92).

13 Como por ejemplo en temas de propiedad intelectual, inversiones o compras públicas.

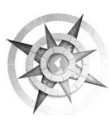


Esta dificultad para cumplir con las normas y los acuerdos establecidos se debe a la falta de desarrollo de una institucionalidad supranacional con competencias necesarias para obligar a los acuerdos y resolver las controversias. Lamentablemente, a los Estados les cuesta ceder su soberanía en estos asuntos, manteniendo esquemas puramente intergubernamentales y bajo reglas de unanimidad $^{14}$, además de normativas regionales débiles y no autónomas que entran en conflicto con las nacionales (Sanahuja, 2007, pp. 84-85; Sanahuja, 2012, p. 28).

Sin embargo, el principal defecto del regionalismo abierto fue su énfasis en las características comerciales que dejó de lado otros componentes importantes dentro del marco de la integración. En especial, el regionalismo abierto no tiene una agenda para

14 A este respecto José Antonio Sanahuja manifiesta: "Como es sabido, el nacionalismo fue discurso fundacional del nuevo poder poscolonial [...] ello ha dado lugar a una paradoja aparentemente irresoluble: por un lado, el nacionalismo y la defensa de la soberanía, como discurso y práctica de la política exterior y doméstica, han sido obstáculos para la construcción de organizaciones regionales fuertes, con competencias propias y soberanía compartida. Por otro lado, la defensa de la soberanía habría sido también ideología común y factor de movilización frente al imperialismo y dominación exterior, y esa tradición emancipadora es un importante elemento constitutivo de las aspiraciones de autonomía de la región (Sanahuja, 2012, p. 22). el desarrollo que brinde una respuesta efectiva a las asimetrías económicas, sociales, territoriales, sectoriales, tecnológicas, productivas, etc., en América Latina. Cuando más, las iniciativas consideraban que los esquemas de integración pueden abordar estos problemas a través del libre juego de las fuerzas del mercado o como parte de un efecto de goteo de las ganancias. Haber apostado solamente por un desarrollo del capital, no permitió una complementariedad equitativa en los esquemas y una verdadera agenda para el desarrollo en AL. Incluso, este tipo de regionalismo plantea una paradoja, pues por un lado se creó con el objeto de hacer frente a los desafíos que le planteaba la globalización, pero al mismo tiempo contribuyó a profundizar ese proceso, al basarse en políticas de liberalización comercial con una baja protección externa (Estay, 1997, pp. 74-75 citado por Alzugaray, 1999; Sanahuja, 2007, p. 84; Sanahuja, 2009, p. 12).

Es por esta razón, que el regionalismo abierto ha atravesado un período de transformaciones desde mediados de la década del 2000. El "consenso" acerca de los beneficios de las políticas neoliberales ha perdido ya su brillo. Todo lo cual ha sido más palpable con el "giro hacia la izquierda" que experimentó América Latina al

42 Apuntes sobre la construcción del concepto de desarrollo en América Latina y su contribución en los procesos de integración en la región
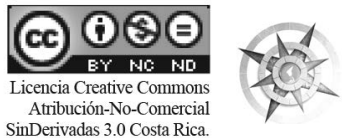
comienzo del siglo XXI y los nuevos esquemas de integración que se han propuesto (Briceño, s.f., p. 23).

\section{El regionalismo post liberal: una propuesta renovada del desarrollo}

$\mathrm{Al}$ igual que en los esquemas de regionalización anteriores, los acontecimientos mundiales y regionales tuvieron un rol importante a la hora de modificar las propuestas de integración. A este respecto, se puede citar el desgaste de la agenda neoliberal, la cual ha sido reemplazada por programas que pretenden más bien administrar los impactos negativos de la globalización. Un ejemplo de esto constituyó el fracaso de los acuerdos de la OMC, en donde se constató que los países del centro no tienen interés por una agenda de desarrollo sino de acceso a los mercados en los países de la periferia. Hecho que fue más notorio tras las protestas antiglobalización de la Conferencia de Seattle (1999), y posteriormente, cuando se rompió con las promesas realizadas en la Ronda de Doha. Además, la crisis financiera del 2008 ha aumentado los escepticismos sobre el libre mercado y la desregulación financiera, incluso entre algunas de las visiones más ortodoxas ${ }^{15}$ (Briceño, s.f., p. 28, Oxfam, 2009, pp. 2-35).

Por otra parte, los magros resultados en el nivel económico de las recetas neoliberales, la vulnerabilidad de la región ante los cambios de la economía internacional ${ }^{16}$, el aumento en las disparidades de la distribución del ingreso en los países e, incluso, la pérdida de interés de Estados Unidos (EEUU) por $\mathrm{AL}^{17}$, crearon un escenario propicio para el resurgimiento de la izquierda en la región. Además, a este fenómeno se sumaron la

15 En el documento elaborado por un alto funcionario del FMI (Repensando la Política Macroeconómica) se realiza un balance del pensamiento del mainstream y afirma que las bases del consenso neoliberal quedaron fuertemente heridas por la crisis del 2007-2008. Por esta razón plantea una nueva alternativa al esquema anterior (Blanchard, 2010 citado por Villadeamigo, 2011).

16 Por ejemplo el impacto que tuvo la crisis asiática sobre la región.

17 "El interés de Estados Unidos por América Latina prácticamente se esfumó después del septiembre de 2001, excepto en materia de comercio y asuntos considerados de seguridad nacional - como la inmigración y el tráfico de drogas o durante los ocasionales ataques de pánico desatados por resultados electorales en países como Bolivia y Venezuela. La "guerra" que emprendió Estados Unidos contra el terrorismo y su subsecuente invasión de Irak simplemente profundizó ese alejamiento, probablemente porque los neoconservadores que operaron como la fuerza ideológica de la administración de George Bush estaban más interesados en afirmar el poderío estadounidense moldeando al Medio Oriente que fortaleciendo las relaciones hemisféricas" (Arditi, 2009, pp. 236-237).

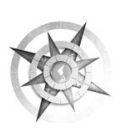
SinDerivadas 3.0 Costa Rica.
Apuntes sobre la construcción del concepto de desarrollo en América Latina y su contribución en los procesos de integración en la región Adriana Montenegro-Braz 
mejoría en términos del intercambio de las materias primas en el ámbito global y un cierto vuelco del comercio regional hacia el área del Asia Pacífico y la Unión Europea (Briceño, s.f., pp. 28-29; Bonilla y Long, 2010, pp. 23-26; CEPAL, 2004, p. 17; Arditi, 2009, pp. 236-237). Esto, en última instancia se tradujo en el regreso de la agenda de desarrollo que tuvo un impacto en la conformación de los nuevos acuerdos regionales e, incluso, en algunos de los acuerdos regionales existentes.

En ese sentido, la suspensión de las negociaciones del ALCA en 2005 dio la pauta de que el consenso sobre el modelo de regionalismo abierto estaba llegando a su fin. Con todo, como lo afirma José Briceño, el fin de la convergencia del modelo neoliberal condujo a dos estrategias diferentes. La primera tomó partido por no modificar los compromisos con el libre comercio, promoción de las inversiones y la inserción en el nivel internacional. Dentro de esta tendencia podemos encontrar a México, Chile, Colombia, Perú y la mayor parte de países centroamericanos, quienes no solo han firmado acuerdos bilaterales con Estados Unidos, sino que han creado o se han mantenido en esquemas de integración de tipo abierto $^{18}$. Por ejemplo, procesos de integración como la CAN o el Sistema de Integración Centroamericana (SICA) siguen manteniendo su énfasis en el aspecto económico; a la par que la creación de la Alianza del Pacífico ${ }^{19}$ permite observar que hay países que aún apuestan por los esquemas de integración con énfasis en el mercado (Briceño, s.f., p. 29).

Por otro lado, existen países que se inclinan por procesos de integración en donde los esquemas comerciales y de inversión no son su prioridad, entre los que se encuentran Argentina, Brasil, Bolivia y Venezuela. Con todo, dentro de este grupo existen esquemas de integración con una visión más estructuralista, en la que se trata de complementar la agenda productiva con la social, como por ejemplo la iniciativas del "Mercosur productivo y social" ${ }^{20}$; y, esque-

18 Hay que tomar con cuidado la clasificación que ofrece Briceño y tomarla como una referencia general, pues si bien países como Colombia, Chile y Perú tienen firmados TLC con EUA, también son parte de esquemas con una visión diferente como UNASUR. Aunque ciertamente este grupo de países apuestan en mayor medida a un bilateralismo asimétrico (Briceño, s.f; Quiliconi, 2013).

19 Entre Colombia, Perú, Chile y México.

20 Para mayor información sobre este tema se puede consultar el documento Mercosul Social y Participativo, disponible en: http://www. spm.gov.br/assuntos/acoes-internacionais/ Articulacao/articulacao-internacional/mercosul/livro-mercosul-social-participativo.pdf,

44 Apuntes sobre la construcción del concepto de desarrollo en América Latina y su contribución en los procesos de integración en la región 
mas de integración con un enfoque más político como la UNASUR y la Comunidad de Estados Latinoamericanos y Caribeños (CELAC), e incluso con una propuesta antiimperialista o "bolivariana" como la Alianza Bolivariana para los Pueblos de Nuestra América, Tratado de Comercio de los Pueblos (ALBA-TCP) (Briceño, s.f., p. 29; Sanahuja, 2012, pp. 31-58).

Ahora bien, se puede decir que los modelos con un componente más político y social en esta etapa de regionalismo posliberal o poshegemónico, comparten algunos enfoques que son: a) Una mayor preocupación por los temas políticos y las agendas de desarrollo (pos Consenso de Washington), y una menor atención a la agenda comercial; b) una mayor participación estatal para guiar los procesos políticos, comerciales y sociales, etc.; c) énfasis en la cooperación sur-sur y, por ende, en los problemas que constituyen el "cuello de botella" de la región, como por ejemplo el desarrollo de la infraestructura regional, reducción de las asimetrías, reducción de la pobreza y la desigualdad, y una creciente preocupación por la seguridad energética;

(Secretaría Geral da Presidencia da República, Ministerio das Relacoes Exteriores, Ministerio do Desenvolvimento Social y Combate a Fome, 2007). d) un mayor peso a la construcción de una agenda positiva de la integración que incluye la creación de instituciones y políticas comunes, así como agendas de coordinación macroeconómica, temas monetarios y finanzas; y, e) una mayor participación de los actores no estatales y generación de fórmulas para una mayor legitimación de los procesos (Sanahuja, 2012, pp. 2-33; Briceño, s.f., p. 29).

En resumen, en la actualidad existe una heterogeneidad y fragmentación de los modelos regionales si se compara con los modelos regionales de la década de los noventa. En ese sentido, existen países que apuestan por los esquemas de regionalismo abierto mientras que otros países se adhieren a los procesos de integración posliberales, modificando sus agendas centradas en lo meramente económico, para considerar aspectos sociales y políticos. Es necesario tomar en consideración que la heterogeneidad de modelos regionales no significa necesariamente una falta de consolidación de los mismos; en ese sentido, no deben ser vistos como "fracasos" sino como "experiencias que se agregan" y que permiten un aprendizaje del proceso regional latinoamericano (véase Carranza, 2014). En ese sentido, el hecho de que se haya incorporado 
nuevamente el tema del desarrollo en las discusiones de los esquemas de integración ha sido uno de los mayores beneficios de esta diversificación.

\section{Conclusiones}

Este estudio se propuso revisar cómo ha ido evolucionando el concepto de desarrollo en América Latina y cómo esta idea permeó, o no, en los modelos de regionalismo. En este sentido, se ha observado que durante los últimos sesenta años de discusión sobre el desarrollo en la región, se han alternado períodos en donde se ha dado más peso al componente político o al económico para lograr un mayor crecimiento. Sin embargo, en el nuevo escenario regional existe una mayor dispersión. Ya no solo conviven diferentes esquemas de integración, sino que además estos difieren respecto a los objetivos que persiguen. Además, se observa también un solapamiento entre las diferentes propuestas, pues en la cuantiosa parte de los estados latinoamericanos pueden convivir dos o más proyectos de integración con distintos enfoques y perspectivas sobre lo que implica el desarrollo.

Esta nueva configuración plantea aspectos positivos, pero también algunos negativos, a los que cabría dar algún tipo de respuesta. Por un lado, se puede decir que uno de los aspectos positivos de la mayor heterogeneidad es justamente cuestionar el modelo neoliberal, plantear ciertas salvaguardas a los efectos de la globalización y regresar a una agenda para el desarrollo en América Latina. Sin embargo, la heterogeneidad se puede transformar fácilmente en un modelo caótico y sin rumbo, que debilite aún más los procesos de integración en la región. Basta con observar los efectos que los TLC con EUA, firmados por Colombia y Perú, tuvieron en la CAN, e incluso los inconvenientes que podría generar la Alianza del Pacífico (AP) o un cambio de tendencia ideológica de algunos países con tendencias progresistas o de izquierda para los procesos de integración del MERCOSUR y UNASUR.

En ese sentido, la falta de una visión compartida entre los países latinoamericanos acerca del papel del Estado (en el nivel externo e interno) y el mercado para generar desarrollo, es lo que ha diversificado aún más los procesos de integración. No obstante, hay que considerar que lo político y lo económico no han estado desligados en ningún momento, no hay procesos de regionalización asépticos, y las supuestas ausencias de lo político sobre lo económico, que se ha dado en algún momento, ciertamente

46 Apuntes sobre la construcción del concepto de desarrollo en América Latina y su contribución en los procesos de integración en la región

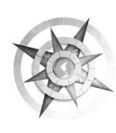


esconden una intencionalidad. Por lo tanto, es necesario replantearse un concepto de desarrollo propio, que permita a los países latinoamericanos, pese a la heterogeneidad y fragmentación, comenzar a hilvanar los diferentes procesos de integración sobre un objetivo común.

\section{Bibliografía}

Alzugaray, C. (1999). El Regionalismo en América Latina y El Caribe en la encrucijada: Las estrategias contrastantes de la integración regional. Revista Globalhoy 17. Consultado el 24 de abril, 2015, en: http://www.gloobal.net/iepala/gloobal/fichas/ficha. php? entidad=Textos\&id=6421\&opcion=documento\#ficha_gloobal

André Gunder, F. (1991). El Desarrollo del Subdesarollo - Un Ensayo Autobiográfico. Caracas: Ed. Nueva Sociedad.

Antxon, M. (2010). La dimensión tecnológica de la globalización. Ponencia presentada en la XII Reunión de Economía Mundial en Santiago de Compostela, mayo de 2010.

Arditi, B. (2009). El giro a la izquierda en América Latina: ¿una política post-liberal?. Revista de Ciencias Sociales Unisinos 45, $\mathrm{N}^{\circ}$.3. Consultado $23 \mathrm{de}$ abril, 2015, en: http://www.redalyc. org/articulo.oa?id=93812729006

Bielschowsky, R. (2009). Sesenta años de la CEPAL: estructuralismo y neoestructuralismo. Revista CEPAL 97, pp.173-194.
Blanchard, Olivier, Giovanni Dell Ariccia y Paolo Mauro. 2010. Rethinking macroeconomic policy. FMI. Consultada 25 de abril, 2015, en:http://jeromevillion.free.fr/ChroniqueSubprimes_Documents/ChroniqueSubprimes_Blanchard2010.pdf

Bonilla, A. y Guillaume, L. (2010). Un nuevo regionalismo sudamericano. Presentación del dossier En Íconos Revista de Ciencias Sociales 38, pp.23-28.

Briceño, J. (s.f.). Del regionalismo abierto al regionalismo poshegemónico en América Latina. Consultado 25 de abril, 2015, en: https://www.academia. edu/8043464/Del_regionalismo_ abierto_al_regionalismo_poshegem\%C3\%B3nico_en_Am\%C3\%A9rica_Latina

Briceño, J. (2003). Las teorías de la integración regional. Venezuela: Universidad de los Andes.

Cardoso, F. H. y Faletto, E. (1971). Dependencia y Desarrollo en América Latina: ensayo de interpretación sociológica. México: Siglo XXI Editores, S. A.

CEPAL. (1951a). Estudio Económico de América Latina 1950 (E/CN.12/217). Santiago de Chile.

CEPAL. (1951b). Estudio Económico de América Latina 1949 (E/CN.12/164/ Rev.1). Santiago de Chile.

CEPAL. (1996). Transformación Productiva con Equidad. Consultado el 25 de abril de 2015, en: http://archivo.cepal.org/pdfs/1991/S9181285.pdf

CEPAL. (2004). Una década de desarrollo social en América Latina. Consultado el 24 de 
abril, 2015, en: http://repositorio.cepal.org/bitstream/handle/11362/2382/ S2004000_es.pdf?sequence $=1$

Dos Santos, T. (1998). La teoría de la dependencia un balance histórico y teórico. Los retos dela globalización. Ensayo en homenaje a Theotonio Dos Santos, editado por Francisco López. Caracas: UNESCO.

Durán, J. y Alessia Lo Turco. (2010). El comercio intrarregional en América Latina: Patrón de especialización y potencial exportador. En Los impactos de la crisis internacional en América Latina, coordinado por María Inés Terra y José Durán. Consultado el 26 de abril, 2015, en: http://www.redmercosur.org/ impactos-de-la-crisis-en-america-latina-hay-margen-para-el-diseno-de-politicas-regionales/ publicacion/171/es/tem_12/

Estay, J. (1997). La integración económica americana: Encuadre general, balance y situación actual. En América Latina en la posguerra fría: tendencias y alternativas, coordinado por Jaime Preciado Coronado, Jaime Estay y John Saxe-Fernández. Guadalajara: Universidad de Guadalajara.

Estay, J. (2000). La ALADI en la Integración Latinoamericana. En La Nueva Integración Económica de América Latina y el Caribe. Balance y Perspectivas en el Cambio de Siglo, coordinado por Jaime Estay. Consultado el 24 de abril, 2015, en: http://www.ieeiunesp.com.br/portal/artigos/La\%20 Aladi\%20en\%20la\%20integracion $\% 20$ latinoamericana $\% 20-\% 20$ Jaime\%20E\%20Estay\%20R.pdf
Hettne, B. (1995). Development theory and the three worlds: Towards an international political economy of development. London: Longman Scietific and Technical.

Ibáñez, J. (2000). El nuevo regionalismo latinoamericano en los años noventa. Revista Electrónica de Estudios Internacionales REEI. Consultado el24 de abril, 2015, en: http://www.reei. org/index.php/revista/num1/agora/ nuevo-regionalismo-latinoamericano-anos-noventa

Lorenzini, M. E. (2014). Pensando desde el Sur: Ideas, aportes y contribuciones teórico-conceptuales de Helio Jaguaribe para comprender las realidades latinoamericanas. En Pensadores del Cono Sur: Los aportes de Jaguaribe, Methol Ferrè, Puig y Tomassini a las Relaciones Internacionales. Documento de Trabajo $\mathrm{N}^{\circ}$. 8, pp.13-33.

Lorenzini, M. E. y Pereyra Doval, M. G. (2013). Revisitando los aportes de las teorías del sur: nexos entre teoría y praxis en Argentina y Brasil. Revista Relaciones Internacionales (22), pp.9-26.

Oxfam. (2009). Promesas vacías iQué ha sido del "desarrollo" en la Ronda de Doha de la OMC? Consultado el 24 de abril, 2015, en: http://www.oxfamintermon.org/es/documentos/20/07/09/ promesas-vacias-que-ha-sido-del-desarrollo-en-ronda-de-doha-de-omc

Palacio, P. (2006). La industrialización nacional y el papel del estado, en el viejo regionalismo latinoamericano y en el nuevo regionalismo abierto americano. Observatorio de la Economía Latinoamericana (57). Consultado el 25

48 Apuntes sobre la construcción del concepto de desarrollo en América Latina y su contribución en los procesos de integración en la región

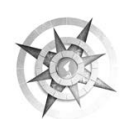
Adriana Montenegro-Braz 
de abril, 2015, en: www.eumed.net/ cursecon/ecolat/la/

Prebisch, R. (1973). Problemas teóricos y prácticos del crecimiento económico. Santiago de Chile: CEPAL.

Prebisch, R. (1996). Cinco etapas de mi pensamiento sobre el desarrollo. El Trimestre Económico (250), $\mathrm{N}^{\circ} .2$, pp.1-20.

Quilliconi, C. (2013). Modelos competitivos de integración en el hemisferio occidental: ¿liderazgo competitivo o negación mútua? Revista CIDOB d`Afers Internacionals N¹02-103, pp. 147-68.

Sanahuja, J. A. (2007). Regionalismo e integración en América Latina: balance y perspectivas. Pensamiento Iberoamericano, pp.75-106. Consultado el 24 de abril, 2015, en: http://dialnet.unirioja.es/servlet/ articulo? codigo $=2872515$

Sanahuja, J. A. (2012). Regionalismo post-liberal y multilateralismo en Sudamérica: El caso de UNASUR. En El Regionalismo Post-Liberal en América Latina y el Caribe: Nuevos actores, nuevos temas, nuevos desafíos. Anuario de la Integración Regional de América Latina y el Gran Caribe, coordinado por Serbin, Andrés, Laneydi Martínez y Aroldo Ramazini Júnior.
Consultado el 24 de abril, 2015, en: http://www.ieei-unesp.com.br/portal/ wp-content/uploads/2012/10/2012. Anuario-CRIES-1.pdf

Secretaría Geral da Presidencia da República, Ministerio das Relacoes Exteriores, Ministerio do Desenvolvimento Social y Combate a Fome. (2007). Mercosul Social y Participativo: Construindo o Mercosul dos povos com democracia e cidadania. Consultada 23 de abril, 2015, en: http://www. spm.gov.br/assuntos/acoes-internacionais/Articulacao/articulacao-internacional/mercosul/livro-mercosul-social-participativo.pdf

Villadeamigo, J. (2011). El cuestionamiento a los enfoques ortodoxos de la economía y las visiones alternativas. El caso de las economías en desarrollo. Consultado el 25 de abril, 2015, en: http://www. uba.ar/archivos_secyt/image/S1\%20 _\%20Documento.PDF

Williamson, J. (1995). Lo que Washington quiere decir cuando se refiere a reformas de las políticas económicas. En Latin American Adjustment: How Much Has Happened?, coordinado por John Williamson, pp.67-86.

Zona Económica.(2015). El Estructuralismo Latinoamericano -Desarrollo. Consultado el 26 de abril, 2015. 